Mathematical Research Letters 8, 641-649 (2001)

\title{
RATIONAL COVARIANTS OF REDUCTIVE GROUPS AND HOMALOIDAL POLYNOMIALS
}

\author{
Hanspeter Kraft and Gerald W. Schwarz
}

\begin{abstract}
Let $G$ be a complex reductive group, $V$ a $G$-module and $f \in \mathcal{O}(V)^{G}$ a nonconstant homogeneous invariant. We investigate relations between the following properties:

- $d f: V \rightarrow V^{*}$ is dominant,

- $f$ is homaloidal, i.e., $d f$ induces a birational map $\mathbb{P}(V) \rightarrow \mathbb{P}\left(V^{*}\right)$,

- $V$ is stable, i.e., the generic $G$-orbit is closed.

If $f$ generates $\mathcal{O}(V)^{G}$, we show that the properties are equivalent, generalizing results of SATO-KIMURA on prehomogeneous vector spaces.
\end{abstract}

\section{Introduction}

Our ground field is $\mathbb{C}$, the field of complex numbers. Throughout this note, $G$ will be a reductive (complex) algebraic group, and $V$ a (finite dimensional algebraic) $G$-module. We denote by $\mathcal{O}(V)$ the algebra of polynomial functions on $V$ and by $\mathcal{O}(V)^{G}$ the subalgebra of $G$-invariant functions. The algebraic quotient (of $V$ by $G$ ) is the morphism $\pi_{V}: V \rightarrow V / / G$ corresponding to the inclusion $\mathcal{O}(V)^{G} \subset \mathcal{O}(V)$. Similar definitions and notation apply to an affine $G$-variety, i.e., an affine variety $X$ equipped with an algebraic action of $G$.

If $f \in \mathcal{O}(V)^{G}$, then the differential

$$
d f: V \rightarrow V^{*}, \quad v \mapsto d f(v):\left(T_{v} V \simeq V\right) \rightarrow \mathbb{C}
$$

is $G$-equivariant, where $V^{*}$ denotes the dual $G$-module. The (schematic) fiber $F:=f^{-1}(f(v))$ is non-singular at $v$ if and only if $d f(v) \neq 0$, in which case Ker $d f(v)=T_{v} F$, the tangent space to $F$ at $v$. Note that if $f$ is homogeneous and $d f$ is dominant, then $d f$ defines a rational map $\Phi_{f}: \mathbb{P}(V) \rightarrow \mathbb{P}\left(V^{*}\right)$ of finite degree. If $\Phi_{f}$ is birational, then $f$ is said to be homaloidal.

We say that $V$ is stable if the set of closed $G$-orbits contains a non-empty open subset of $V$. The main results of this paper are the following.

Theorem A. Let $f \in \mathcal{O}(V)^{G}$ be a homogeneous invariant. If the differential $d f: V \rightarrow V^{*}$ is dominant, then $V$ is a stable $G$-module.

Received April 5, 2001

Both authors thank the Mathematical Sciences Research Institute and the Erwin Schrödinger Institute for hospitality, and they thank the SNF and NSF, respectively, for partial support. 
Theorem B. Assume that the invariant ring $\mathcal{O}(V)^{G}$ is generated by a homogeneous function $f$. Then the following are equivalent:

(i) The differential $d f: V \rightarrow V^{*}$ is dominant;

(ii) The differential df induces a birational map $\Phi_{f}: \mathbb{P}(V) \rightarrow \mathbb{P}\left(V^{*}\right)$, i.e., $f$ is homaloidal;

(iii) The $G$-module $V$ is stable.

A crucial step in the proof of Theorem B is Proposition C, below, which is interesting in its own right. In a way it gives conditions for LunA's Slice Theorem ([Lu73], see also [Sl89]) to hold "generically." Recall that a $G$-equivariant morphism $\varphi: X \rightarrow Y$ is excellent if the induced morphism $\bar{\varphi}: X / / G \rightarrow Y / / G$ is étale and the following diagram is cartesian:

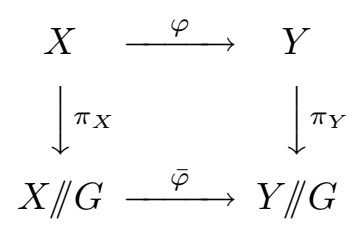

In particular, excellent morphisms are étale. A subset $S$ of an affine $G$-variety $X$ is saturated if $S=\pi_{X}^{-1}\left(\pi_{X}(S)\right)$.

Proposition C. Let $X$ and $Y$ be two smooth affine $G$-varieties of the same dimension and let $\varphi: X \rightarrow Y$ be a G-equivariant dominant morphism. Assume that the generic fibers (see $\S 1$ ) of $\pi_{X}$ and $\pi_{Y}$ are $G$-isomorphic. Then there is a non-empty saturated open set $U \subset Y$ such that the induced morphism $\left.\varphi\right|_{\varphi^{-1}(U)}: \varphi^{-1}(U) \rightarrow U$ is excellent.

In the special case of a $G$-module and its dual the assumption on the generic fibers is automatically fulfilled.

Proposition D. Let $\varphi: V \rightarrow V^{*}$ be a G-equivariant dominant morphism. Then the generic fibers of $\pi_{V}$ and $\pi_{V^{*}}$ are isomorphic.

The following relations between $V$ and $V^{*}$ come in handy:

Proposition E. Let $V$ be a $G$-module. Then

(1) $V$ is stable if and only if $V^{*}$ is stable.

(2) The principal isotropy groups (see $§ 1)$ of $V$ and $V^{*}$ are the same.

Outline of the paper. In $\S 1$ we state and prove a generalization (Proposition 1) of Proposition C, and we prove Propositions D and E and Theorem A. In $\S 2$ we consider the special case of $G$-modules with one-dimensional quotient, and we give the proof of Theorem B, which generalizes results obtained by SATOKIMURA [SaK77] for prehomogeneous vector spaces. In $\S 3$ we show how to obtain the Sato-Kimura results from Theorem B. 


\section{$\S 1$. Dominant Covariants}

Let $X$ be an affine $G$-variety. Note that any fiber $F$ of $\pi_{X}: X \rightarrow X / / G$ is without invariants in the sense that $\mathcal{O}(F)^{G}=\mathbb{C}$. We say that $F$ is a generic fiber of $\pi_{X}$ (or of $X$ ) if there is a nonempty open subset $Z \subset X / / G$ such that $\pi_{X}^{-1}(Z) \rightarrow Z$ is a $G$-fiber bundle with fiber $F$ (in the étale topology). LunA's slice theorem [Lu73] shows that any smooth affine $G$-variety has a generic fiber $F$. Moreover, $F$ has the form $G *^{H} W$ where $H \subset G$ is reductive and $W$ is an $H$-module without invariants. In particular, $F$ is smooth and $G$ acts transitively on the irreducible components of $F$. The subgroup $H$ (or any $G$-conjugate of $H$ ) is called a principal isotropy group of $X$. A nonsmooth affine $G$-variety may not have a generic fiber.

For completeness, we add the following well-known result:

Lemma 1. Let $\varphi: F \rightarrow F^{\prime}$ be a dominant $G$-equivariant morphism where $F$ and $F^{\prime}$ are $G$-isomorphic affine varieties without invariants. Then $\varphi$ is an isomorphism.

Proof. Since $\varphi$ is dominant, we have a $G$-linear inclusion $\varphi^{*}: \mathcal{O}\left(F^{\prime}\right) \hookrightarrow \mathcal{O}(F)$. By assumption, the isotypic components of $\mathcal{O}(F)$ and $\mathcal{O}\left(F^{\prime}\right)$ are finite dimensional of the same dimension. Thus $\varphi^{*}$ is an isomorphism.

The next result is a generalization of Proposition $\mathrm{C}$ of the introduction.

Proposition 1. Let $X$ and $Y$ be irreducible affine $G$-varieties of the same dimension which have generic fibers $F_{X}$ and $F_{Y}$, respectively. Assume that $F_{X}$ and $F_{Y}$ are $G$-isomorphic and that $G$ acts transitively on the irreducible components of $F_{X}$. If $\varphi: X \rightarrow Y$ is a dominant $G$-equivariant morphism, then there is a non-empty saturated open set $U \subset Y$ such that $\varphi$ is excellent on $\varphi^{-1}(U)$.

Proof. It suffices to find a (non-empty) saturated open set $X^{\prime} \subset X$ such that $\left.\varphi\right|_{X^{\prime}}: X^{\prime} \rightarrow Y$ is excellent, since every such $X^{\prime}$ contains a dense subset of the form $\varphi^{-1}(U)$ where $U \subset Y$ is open and saturated. Therefore, we can assume that $\pi_{X}: X \rightarrow X / / G$ and $\pi_{Y}: Y \rightarrow Y / / G$ are both fiber bundles over smooth bases with fibers $F_{X}$ and $F_{Y}$, respectively, and that $\bar{\varphi}: X / / G \rightarrow Y / / G$ is dominant. Shrinking further we can assume that $\bar{\varphi}$ is étale.

Let $X_{0} \subset X$ be the (non-empty) $G$-stable open set where $\varphi$ is smooth. If a fiber $F$ of $\pi_{X}$ meets $X_{0}$, then $\overline{\varphi(F)}$ has the same dimension as $F$, hence contains an irreducible component of a fiber $F^{\prime}$ of $\pi_{Y}$. Since $G$ acts transitively on the irreducible components of the fibers, we get that $\overline{\varphi(F)}=F^{\prime}$, and Lemma 1 shows that $\varphi$ induces an isomorphism $F \stackrel{\sim}{\rightarrow} F^{\prime}$.

The argument above shows that we can assume that every fiber of $\pi_{X}$ is mapped isomorphically onto a fiber of $\pi_{Y}$. Thus we obtain a map $\tilde{\varphi}: X \rightarrow$ $X / / G \times_{Y / / G} Y$ of fiber bundles over $X / / G$ inducing isomorphisms on the fibers. The following lemma shows that $\tilde{\varphi}$ is an isomorphism, i.e., that $\varphi$ is excellent. 
The lemma below is certainly well-known. Since we could not find a suitable reference, we include a short proof.

Lemma 2. Let $p: X \rightarrow S$ and $q: Y \rightarrow S$ be two fiber bundles with fiber $F$ and let $\varphi: X \rightarrow Y$ be a morphism of fiber bundles (i.e., $q \circ \varphi=p$ ) inducing isomorphisms $\varphi_{s}: X_{s} \stackrel{\sim}{\rightarrow} Y_{s}$ on the fibers for all $s \in S$. Then $\varphi$ is an isomorphism.

Proof. (a) Let us first assume that $\varphi$ is a finite morphism and let $x \in X, y:=$ $\varphi(x) \in Y$ and $s:=p(x)=q(y)$. Then $\varphi^{*}: \mathcal{O}_{Y, y} \rightarrow \mathcal{O}_{X, x}$ is finite and injective and induces, by assumption, an isomorphism $\mathcal{O}_{Y, y} / \mathfrak{m}_{s} \mathcal{O}_{Y, y} \stackrel{\sim}{\rightarrow} \mathcal{O}_{X, x} / \mathfrak{m}_{s} \mathcal{O}_{X, x}$. It follows that $\mathcal{O}_{X, x}=\mathcal{O}_{Y, y}+\mathfrak{m}_{y} \mathcal{O}_{X, x}$ and so $\mathcal{O}_{X, x}=\mathcal{O}_{Y, y}$ by NAKAYAMA's Lemma. Hence $\varphi$ is an isomorphism.

(b) Next we remark that the lemma also holds if $Y$ is normal, since every bijective morphism onto a normal variety is an isomorphism, by ZARISKI's Main Theorem (see [Mu88, Ch. 3, §9]).

(c) In general, let $\tilde{X} \rightarrow X$ be the normalization of $X$ and $\tilde{S} \rightarrow S$ the normalization of $S$. We obtain a commutative diagram

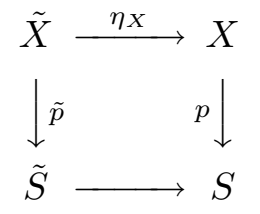

where all horizontal maps are finite and where $\tilde{p}$ is a fiber bundle with fiber $\tilde{F}$, the normalization of $F$. A similar diagram is obtained for $Y$. Moreover, $\varphi$ induces a fiber bundle morphism $\tilde{\varphi}: \tilde{X} \rightarrow \tilde{Y}$ which is again an isomorphism on the fibers. It now follows from (b) that $\tilde{\varphi}$ is an isomorphism:

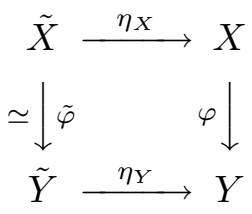

Therefore, $\varphi$ is finite and the lemma follows from (a).

In order to prove Propositions D and E of the introduction, we use the following well-known lemma:

Lemma 3. Let $G$ be a connected reductive group. Then there exists an automorphism $\tau: G \stackrel{\sim}{\rightarrow} G$ with the following property: If $\rho: G \rightarrow \mathrm{GL}(V)$ is a representation of $G$, then $\rho \circ \tau: G \rightarrow \mathrm{GL}(V)$ is equivalent to the dual representation $\rho^{*}: G \rightarrow \mathrm{GL}\left(V^{*}\right)$.

As a consequence, we see that for a connected reductive group $G$ any covariant $\varphi: V \rightarrow W$ can be regarded as a covariant $\varphi^{*}: V^{*} \rightarrow W^{*}$ by simply changing the action of $G$ by the automorphism $\tau$. Moreover, the quotient map $\pi_{V}: V \rightarrow V / / G$ can be identified with the quotient map $\pi_{V^{*}}: V^{*} \rightarrow V^{*} / / G$. 
Remark. It is essential in the lemma above that $G$ be connected. In fact, the smallest Mathieu group $M_{11}$ has a non self-dual representation $W$ of dimension 10, but $M_{11}$ has no outer automorphism. (This example was shown to us by Guralnick.) We do not know if the invariant rings of $W$ and $W^{*}$ are isomorphic.

Proof of Proposition D. Let $\varphi: V \rightarrow V^{*}$ be a dominant covariant. We want to show that the generic fibers of $\pi_{V}$ and $\pi_{V^{*}}$ are isomorphic. We first assume that $G$ is connected. We have seen above that $\varphi$ can be regarded as a covariant $\varphi^{*}: V^{*} \rightarrow V$, and the composition $\varphi^{*} \circ \varphi: V \rightarrow V$ is a dominant covariant. It follows from Proposition 1 that for a generic fiber $F$ of $V$ the image $F^{\prime}:=$ $\varphi^{*}(\varphi(F))$ is also a generic fiber of $V$, and the composition $F \rightarrow \overline{\varphi(F)} \rightarrow F^{\prime}$ is an isomorphism. Hence $F \stackrel{\sim}{\rightarrow} \overline{\varphi(F)}$. Since the generic fibers of $V$ and $V^{*}$ are irreducible and of the same dimension, it follows that $\overline{\varphi(F)}$ is a generic fiber $F^{*}$ of $V^{*}$ and that $F \stackrel{\sim}{\rightarrow} F^{*}$.

In case of a general reductive group $G$ we can apply Propositions $\mathrm{C}$ and D to $G^{0}$ and find a $G^{0}$-saturated open set $U \subset Y$ such that $\varphi$ is excellent on $\varphi^{-1}(U)$ with respect to the action of $G^{0}$. Replacing $U$ be $G U$ we may assume that $U$ is $G$-saturated. In the following diagram

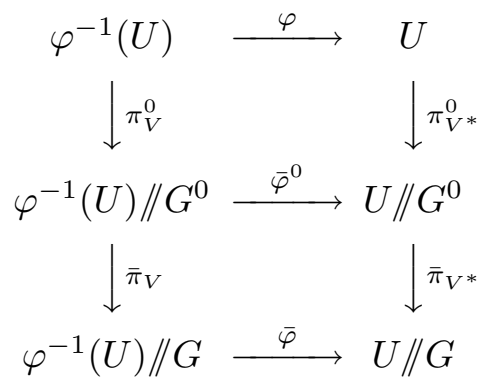

the upper square is cartesian, the maps $\pi_{V}^{0}$ and $\pi_{V^{*}}^{0}$ are the quotients of the $G^{0}$ action, and the maps $\bar{\pi}_{V}$ and $\bar{\pi}_{V^{*}}$ are quotients by the finite group $\bar{G}:=G / G^{0}$. It suffices to show that the generic fibers of $\bar{\pi}_{V}$ and $\bar{\pi}_{V^{*}}$ have the same number of elements, or, equivalently, that the generic fibers of $\pi_{V}$ and $\pi_{V^{*}}$ have the same number of components.

Let $G^{\prime}$ denote the kernel of the action of $G$ on $V / / G^{0}$. Then the number $n_{V}$ of components of the generic fiber of $\pi_{V}$ equals $\left[G: G^{\prime}\right]$. The following lemma shows that $n_{V}=n_{V^{*}}$, proving the proposition.

Lemma 4. Let $N \subset G$ be a normal subgroup, and let $W$ be a $G$-module. Then $\left(W^{N}\right)^{*}$ and $\left(W^{*}\right)^{N}$ are isomorphic $G / N$-modules. In particular, the kernels of the actions of $G$ on $W^{N}$ and $\left(W^{*}\right)^{N}$ are the same.

Proof. One easily reduces to the case that $W$ is an irreducible $G$-module. Since $W^{N}$ is $G$-stable, either $N$ acts trivially on $W$, or $N$ acts nontrivially on $W$ and $W^{N}=(0)$. But $W^{*}$ is also an irreducible $G$-module, and $N$ acts trivially on $W^{*}$ 
if and only if it acts trivially on $W$. Thus, $\left(W^{N}\right)^{*}$ and $\left(W^{*}\right)^{N}$ are either both zero or $W^{*}$.

Proof of Proposition E. Let $V$ be a $G$-module, and let $V_{0}$ denote $V$ considered as a $G^{0}$-module. Then $V$ is stable if and only if $V_{0}$ is stable. Since $V_{0}$ is stable if and only if the generic fiber of $\pi_{V_{0}}$ is an orbit, the comments following Lemma 3 show that $V_{0}$ is stable if and only if $V_{0}^{*}$ is stable. Thus stability of $V$ and $V^{*}$ are equivalent.

Let $H$ be a principal isotropy group of $V$, and let $N$ denote $N_{G}(H) / H$. By the Luna-Richardson Theorem [LuR79], the restriction map $\mathcal{O}(V)^{G} \rightarrow \mathcal{O}\left(V^{H}\right)^{N}$ is an isomorphism where the action of $N$ on $V^{H}$ has trivial principal isotropy groups (hence is stable). The action of $N$ on $\left(V^{*}\right)^{H} \simeq\left(V^{H}\right)^{*}$ is then also stable with finite principal isotropy groups. By [Lu75], $\mathcal{O}\left(V^{*}\right)^{G} \rightarrow \mathcal{O}\left(\left(V^{*}\right)^{H}\right)^{N}$ is finite, and every closed $N$-orbit in $\left(V^{*}\right)^{H}$ lies in a closed $G$-orbit in $V^{*}$. Since $\mathcal{O}\left(\left(V^{*}\right)^{H}\right)^{N}$ and $\mathcal{O}\left(V^{*}\right)^{G}$ have the same dimension (= $\left.\operatorname{dim} V / / G\right)$, some closed $N$ orbit in $\left(V^{*}\right)^{H}$ intersects the closed orbit of a generic fiber of $V^{*}$. It follows that $H \subset H^{*}$, where $H^{*}$ is a principal isotropy group of $V^{*}$. Repeating the argument for $V^{*}$ shows that $H^{*}$ is contained in a conjugate of $H$, hence $H=H^{*}$.

We now give the proof of Theorem A of the introduction.

Proof of Theorem $A$. Let $\varphi: V \rightarrow V^{*}$ be a covariant. For any point $v \in V$, the derivative $d \varphi(v): T_{v} V \rightarrow T_{\varphi(v)} V^{*} \simeq V^{*}$ gives a bilinear form $\beta_{v}$ on $T_{v} V \simeq V$, namely, $\beta_{v}\left(w_{1}, w_{2}\right):=d \varphi(v)\left(w_{1}\right)\left(w_{2}\right)$. Since $\varphi$ is a covariant, $\beta_{v}$ is $G_{v}$-invariant, and $\beta_{v}$ is non-degenerate precisely at the points $v$ where $\varphi$ is étale. Now assume that $f \in \mathcal{O}(V)^{G}$ and $\varphi:=d f$. Then the form $\beta_{v}$ is the Hessian of $f$ and is symmetric. If $\varphi$ is dominant, Propositions $\mathrm{C}$ and $\mathrm{D}$ give us an open $G$-saturated subset $U \subset V^{*}$ such that $\left.\varphi\right|_{\varphi^{-1}(U)}: \varphi^{-1}(U) \rightarrow U$ is excellent, and we may assume that $U$ and $\varphi^{-1}(U)$ are affine. The following result of LunA (see [Lu72], [Lu73]) shows that the action of $G$ on $\varphi^{-1}(U)$ is stable, proving Theorem A.

Theorem (Luna). Let $X$ be a smooth affine G-variety. Assume that for each $x \in X$ there is a $G_{x}$-invariant non-degenerate symmetric bilinear form on the tangent space $T_{x} X$. Then $X$ is stable.

\section{$\S 2$. One-dimensional quotients}

Let $V$ be a $G$-module such that the invariant $\operatorname{ring} \mathcal{O}(V)^{G}$ is generated by the homogeneous function $f$ of degree $d$. Then $\mathcal{O}\left(V^{*}\right)^{G}$ is also generated by a homogeneous function $f^{*}$ of degree $d$.

Proposition 2. Let $V, G, f$ and $f^{*}$ be as above and let $G v=\overline{G v}$ be a closed orbit in $V$. Then

(1) $f^{*} \circ d f$ is a non-zero multiple of $f^{d-1}$.

(2) $d f^{*}(d f(v))=c_{v} v$ for some non-zero $c_{v} \in \mathbb{C}$.

(3) If $V$ is stable, then $d f$ is dominant. 
Proof. We may choose an isomorphism $V \simeq \mathbb{C}^{n}$ such that $G \subset \mathrm{GL}_{n}(\mathbb{C})$ is the complexification of a compact subgroup $K \subset \mathrm{U}_{n}(\mathbb{C})$. Let ( , ) denote the standard hermitian inner product on $\mathbb{C}^{n}$. Then Kempf-Ness theory ([KeN79], cf. [Sch89]) tells us the following: Let $v \in V$. Then

$(*) \quad T_{v}(G v) \perp v$ if and only if $G v$ is a closed orbit and $(v, v)=\inf _{g \in G}(g v, g v)$.

Assume that $V$ is stable, and choose $0 \neq v \in V$ such that $G v$ is closed. We may assume that $(g v, g v)$ achieves its minimum at $v$. If $d f(v)=0$, then $d f$ vanishes on $G(\mathbb{C} v)$ which is dense in $V$. Thus $d f \equiv 0$, which is impossible. Let $\lambda$ denote the linear form $d f(v) \neq 0$. Then $T_{v}(G v)=\operatorname{Ker} \lambda$ has dimension $n-1$, and so $\lambda(w)=c(w, v)$ for all $w \in V$ and a suitable $c \in \mathbb{C}$. Considered as an element of $V^{* *}, v$ lies in the annihilator of $T_{\lambda}(G \lambda)$ : if $A \in \mathfrak{g}$, then $(A \lambda)(v)=\lambda(-A v)=$ $c(-A v, v) \in(\mathfrak{g} v, v)=\{0\}$. But this shows that $\lambda \perp T_{\lambda}(G \lambda)$ (using the induced hermitian inner product on $\left.V^{*}\right)$. Thus $G \lambda$ is a closed orbit in $V^{*}$ and $\lambda \in G \lambda$ is a point of minimal norm. Hence $f^{*}(\lambda) \neq 0$, giving (1). Since $V^{*}$ is also stable, we may apply the reasoning above to $v^{\prime}:=d f^{*}(\lambda) \in V^{* *}$. We obtain that $v^{\prime}$ generates the annihilator of $T_{\lambda}(G \lambda)$, so that $v^{\prime}$ is a non-zero multiple of $v \in V^{* *}$. We have (2), and (3) follows since $G(\mathbb{C} \lambda)$ is dense in $V^{*}$.

In general, let $H$ be a principal isotropy group of $V$. Then the action of $N:=N_{G}(H) / H$ on $V^{H}$ is stable, and by the Luna-Richardson Theorem [LuR79], the restriction $\bar{f}$ of $f$ to $V^{H}$ generates the invariant $\operatorname{ring} \mathcal{O}\left(V^{H}\right)^{N}$. For $v \in V^{H}$, we have $d f(v) \in\left(V^{*}\right)^{H}$, and using the canonical isomorphism $\left(V^{*}\right)^{H} \simeq\left(V^{H}\right)^{*}$ this linear function identifies with $\left.d f(v)\right|_{V^{H}}=d \bar{f}(v)$. Proposition E shows that $H$ is also the principal isotropy group of $V^{*}$. Hence $\bar{f}^{*}=\left.f^{*}\right|_{V^{* H}}$ generates the invariant ring $\mathcal{O}\left(V^{* H}\right)^{N}$. By the first part of our proof, $f^{*}(d f(v))=\bar{f}^{*}(d \bar{f}(v)) \neq$ 0 and $d f^{*}(d f(v))=d \bar{f}^{*}(d \bar{f}(v))$ is a multiple of $v$, so we have (1) and (2).

Corollary 1. Let $V, f$ and $f^{*}$ be as above. If $d f: V \rightarrow V^{*}$ is dominant, then $d f^{*} \circ d f=c f^{d-2} \operatorname{Id}_{V}$ for a non-zero constant $c \in \mathbb{C}$.

Proof. Since $d f$ is dominant, $V$ is stable by Proposition 1 and $d f^{*}(d f(v))$ is a nonzero multiple of $v$ for every $v$ not in the nullcone $\mathcal{N}_{V}=f^{-1}(0)$ of $V$. Since $G \times \mathbb{C}^{*}$ has a dense orbit in $V$ and since $d f^{*} \circ d f$ is $G$-equivariant and homogeneous, $d f^{*} \circ d f(w)=h(w) w$ for all $w \in V$, where $h(w)$ is a nonzero homogeneous polynomial of degree $d(d-2)$. Clearly, $h$ is $G$-invariant, hence it has the given form.

We now give the proof of Theorem B of the introduction.

Proof of Theorem $B$. If $d f$ induces a birational map $\Phi_{f}: \mathbb{P}(V) \rightarrow \mathbb{P}\left(V^{*}\right)$, then $d f$ is dominant, and conversely by Corollary 1. Dominance of $d f$ and stability of $V$ are equivalent by Proposition 2 and Theorem A. 
Examples. It is easy to find cases where $d f$ is not dominant. Start with any $G$-module $V$ with a one-dimensional quotient $f: V \rightarrow \mathbb{C}$, let $H$ be a reductive group and $W$ a nonzero $H$-module without invariants. Then $\tilde{V}:=V \oplus W$ is a $\tilde{G}:=G \times H$-module with a one-dimensional quotient given by $\tilde{f}:(v, w) \mapsto f(v)$. Clearly $d \tilde{f}(\tilde{V}) \subset V$, so $d f$ is not dominant.

\section{$\S 3$. Prehomogeneous vector spaces}

Let $V$ be a prehomogeneous $G$-module and $\chi$ a character of $G$. A function $f: V \rightarrow \mathbb{C}$ is called a semi-invariant (with character $\chi$ ) if $f(g v)=\chi(g) f(v)$ for all $g \in G$. It is easy to see that semi-invariants have to be homogeneous functions. Note that the subalgebra $\mathbb{C}[f] \subset \mathcal{O}(V)$ is the invariant ring under $G_{0}:=\operatorname{Ker} \chi$. In fact, $f$ is $G_{0}$-invariant and the fibers of the restriction of $f$ to the dense orbit $G v$ are exactly the $G_{0}$-orbits.

We say that $V$ is regular if there is a semi-invariant $f$ whose Hessian does not vanish identically; equivalently (by Theorem B), $f$ is homaloidal. The following result is essentially contained in the work [SaK77] of SATO-KimuRA.

Theorem 3. Let $V$ be prehomogeneous, and let $v \in V$ such that $G v$ is dense in $V$. Then the following are equivalent:

(1) $V$ is regular.

(2) There is a semi-invariant $f$ with character $\chi$ such that $V$ is a stable $G_{0}$-module, where $G_{0}:=\operatorname{Ker} \chi$ (a reductive group).

(3) The isotropy group $G_{v}$ is reductive.

(4) The complement of $G v$ is a hypersurface.

If these conditions hold then a semi-invariant $f$ is homaloidal if and only if its zero set is the complement of the dense orbit Gv.

Proof. Given a semi-invariant $f$, let $\chi_{f}$ denote the corresponding character. If $V$ is regular, then we have a semi-invariant $f$ such that $d f: V \rightarrow V^{*}$ is dominant, and an application of Theorem A (or B) shows that $V$ is a stable $\left(G_{0}:=\operatorname{Ker} \chi_{f}\right)$ module. Thus (1) implies (2). If $G_{0}$ and $f$ are as in (2), then the isotropy group $\left(G_{0}\right)_{v}$ is reductive. Since $f(v) \neq 0$, we have $G_{v}=\left(G_{0}\right)_{v}$, giving (3). If (3), then $G v \simeq G / G_{v}$ is affine, which forces the complement $V \backslash G v$ to be a hypersurface, giving (4). If $S:=V \backslash G v$ is a hypersurface, let $f$ be a function defining $S$. Then $f$ is a homogeneous semi-invariant, and the fibers of $f: V \rightarrow \mathbb{C}$ over $\mathbb{C} \backslash\{0\}$ are the $\left(G_{0}:=\operatorname{Ker} \chi_{f}\right)$-orbits in $G v$. Since all of these orbits have dimension $\operatorname{dim} V-1$, they are all closed. Hence the action of $G_{0}$ on $V$ is stable, and $V$ is regular by Theorem B. Thus (4) implies (1).

Let $f$ be a semi-invariant. If $f^{-1}(0)=S:=V \backslash G v$, we have shown that $f$ is homaloidal. Suppose that $f^{-1}(0) \neq S$. Then there is an irreducible component $S^{\prime}$ of $S$ such that $\left.f\right|_{S^{\prime}}: S^{\prime} \rightarrow \mathbb{C}$ is dominant. It follows that the generic fiber of $f$ consists of a $\left(G_{0}:=\operatorname{Ker} \chi_{f}\right)$-orbit in $G v$, together with at least one other orbit. Hence $V$ is not a stable $G_{0}$-module, and $f$ is not homaloidal. 
Remark. The last statement of the theorem above proves a very special case of a conjecture by DOLGACHEV claiming that a homogeneous polynomial $f$ with prime decomposition $\prod_{i} f_{i}^{n_{i}}$ is homaloidal if and only if $\prod_{i} f_{i}$ is homaloidal (see [Do00], end of section 3).

Example. Let $G=\mathrm{SL}_{2} \times\left(\mathbb{C}^{*}\right)^{3}$ and $V=\left(\mathbb{C}^{2}\right)^{3}$. The group $\mathrm{SL}_{2}$ acts on each copy of $\mathbb{C}^{2}$ in the standard way, and $\left(t_{1}, t_{2}, t_{3}\right) \in\left(\mathbb{C}^{*}\right)^{3}$ sends $\left(v_{1}, v_{2}, v_{3}\right) \in V$ to $\left(t_{1} v_{1}, t_{2} v_{2}, t_{3} v_{3}\right)$. Clearly $V$ is a prehomogeneous $G$-module. Let $f_{i j}\left(v_{1}, v_{2}, v_{3}\right)$ denote the determinant $\left\langle v_{i}, v_{j}\right\rangle, 1 \leq i<j \leq 3$. Then the semi-invariants are the products of the $f_{i j}$, and a semi-invariant is homaloidal if and only if it has each of $f_{12}, f_{13}$ and $f_{23}$ as a factor.

\section{References}

[Do00] Dolgachev, I.V., Polar Cremona transformations, Michigan Math. J. 48 (2000), 191202.

[KeN79] Kempf, G.; Ness, L., The length of a vector in a representation space, Algebraic Geometry, Lecture Notes in Math., vol. 732, Springer Verlag, Berlin-Heidleberg-New York, 1979, 233-243.

[Lu72] Luna, D., Sur les orbites fermées des groups algébriques réductifs, Invent. Math. 16 (1972), 1-5.

[Lu73] 는 Slices étales, Bull. Soc. Math. France, Mémoire 33 (1973), 81-105.

[Lu75] _ Adhérences d'orbite et invariants, Invent. Math. 29 (1975), 231-238.

[LuR79] Luna, D.; Richardson, R. W., A generalization of the Chevalley restriction theorem, Duke Math. J. 46 (1979), 487-496.

[Mu88] Mumford, D., The Red Book of Varieties and Schemes, Lecture Notes in Math. vol. 1358, Springer Verlag, Berlin-Heidleberg-New York, 1988.

[SaK77] Sato, M.; Kimura, T., A classification of irreducible prehomogeneous vector spaces and their relative invariants, Nagoya Math. J. 65 (1977), 1-155.

[Sch89] Schwarz, G. W., The topology of algebraic quotients, Topological Methods in Algebraic Transformation Groups (H. Kraft et al., eds.), Progr. Math., vol. 80, Birkhäuser Verlag, Basel-Boston, 1989, 135-152.

[Sl89] Slodowy, P., Der Scheibensatz für algebraische Transformationsgruppen, Algebraische Transformationsgruppen und Invariantentheorie (H. Kraft, P. Slodowy, T. A. Springer, eds.), DMV-Seminar, vol. 13, Birkhäuser Verlag, Basel-Boston, 1989, 89113.

Mathematisches Institut Der Universität Basel, Rheinsprung 21, CH-4051 Basel, SWITZERLAND.

E-mail address: kraft@math.unibas.ch

Department of Mathematics, Brandeis University, PO Box 549110, Waltham, MA 02454-9110, USA.

E-mail address: schwarz@brandeis.edu 